\title{
Size shrinking of composite bosons for increasing density in the BCS to Bose-Einstein crossover
}

\author{
N. Andrenacci, P. Pieri, and G.C. Strinati \\ Dipartimento di Matematica e Fisica, Sezione INFM Università di Camerino, I-62032 Camerino, Italy
}

(August 11, 2018)

\begin{abstract}
We consider a system of fermions in the continuum case at zero temperature, in the strong-coupling limit of a short-range attraction when composite bosons form as bound-fermion pairs. We examine the density dependence of the size of the composite bosons at leading order in the density ("dilute limit"), and show on general physical grounds that this size should decrease with increasing density, both in three and two dimensions. We then compare with the analytic zero-temperature mean-field solution, which indeed exhibits the size shrinking of the composite bosons both in three and two dimensions. We argue, nonetheless, that the two-dimensional mean-field solution is not consistent with our general result in the "dilute limit", to the extent that mean field treats the scattering between composite bosons in the Born approximation which is known to break down at low energy in two dimensions.
\end{abstract}

PACS numbers: 74.20.Fg, 74.25.-q, 74.20.Mn, 74.20.-z

\section{INTRODUCTION}

The BCS to Bose-Einstein (BE) crossover can be regarded as an evolution from large overlapping Cooper pairs (BCS limit) to small nonoverlapping (composite) bosons (BE limit). At zero temperature, this crossover has thus been characterized in the continuum case in terms of the correlation length $\xi_{\text {pair }}$ for pairs of oppositespin fermions (in units of $k_{F}^{-1}$, where $k_{F}$ is the Fermi wave vector).

It was found that, by reducing the strength of the fermionic attraction at fixed density, $\xi_{\text {pair }}$ increases monotonically from its strong-coupling (BE) limit (equal to the bound-state radius of the associated two-body problem) to the Pippard value in the weak-coupling (BCS) limit.

It was emphasized recently that, as the particle density increases for fixed value of the potential strength, the system also evolves from the BE limit toward the crossover region, eventually reaching the BCS limit (provided certain conditions on the fermionic attraction are fulfilled).

In this context, one would naively expect $\xi_{\text {pair }}$ to increase monotonically, too, when evolving from the $\mathrm{BE}$ limit toward the crossover region by increasing the particle density. A more careful analysis, however, shows that $\xi_{\text {pair }}$ should actually decrease with increasing density starting from the BE limit for small density ("dilute limit"). Consideration of this effect, which originates from the repulsive interaction between the composite bosons, is the main purpose of this paper.

The composite bosons (which form as bound pairs from the constituent fermions in the strong-coupling limit) mutually interact ria a residual repulsive interaction due to Pauli principle. 3 This repulsive interaction, in turn, affects the size of the composite bosons. Specifically, one expects on physical grounds this interaction to reduce the size of the composite bosons (with respect to the boundstate radius of a composite boson in isolation), insofar as the repulsive interaction itself decreases for decreasing size of the composite bosons. A new equilibrium size for the composite bosons thus results when combining the effect of the repulsive interaction with the internal energy of a composite boson, the new equilibrium size being smaller than the original size of a composite boson in isolation.

We shall implement this qualitative idea by minimizing the expression of (twice) the fermionic chemical potential $2 \mu=-\epsilon+\mu_{B}$ with respect of the size $\xi_{\text {pair }}$ of a composite boson, where $\epsilon$ represents the internal energy of a composite boson (to be defined below) and $\mu_{B}$ is the bosonic chemical potential determined by the mutual repulsion between the composite bosons. 5 Both $\epsilon$ and $\mu_{B}$ are, in fact, functions of $\xi_{\text {pair }}$; in addition, $\mu_{B}$ depends on the density $n$.

We shall explicitly verify that the size-shrinking effect is borne out by the analytic zero-temperature mean-field solufion for a point-contact interaction both in three 6 and twol dimensions. In two dimensions, however, the meanfield density dependence of $\xi_{\text {pair }}$ does not agree with what expected in the "dilute limit". We attribute this difference to the poor treatment of the bosonic scattering within the zero-temperature mean field, which rests on the Born approximation as the form of the mean-field bosonic chemical potential implies. Since the Born approximation in two dimensions is known to fail at low energy (which, in turn, corresponds to the "dilute limit" of the Bose gas), the effect of the bosonic interaction on $\xi_{\text {pair }}$ cannot be properly treated within the Born approximation.

We will therefore argue that a proper treatment of the residual bosonic interaction (over and above the Born approximation), along the lines recently developed for the three-dimensional case, 8 should be especially relevant to the two-dimensional case.

We have focused in this paper on the composite-boson regime of the BCS-BE crossprer problem, following previous work on this problem 8 which has emphasized the 
importance of approaching the bosonization in reverse, that is, starting from the $\mathrm{BE}$ and evolving toward the BCS region. Although this reverse approach is not usually adopted in the literature, we prefer it over the conventional $\mathrm{BCS}$ to $\mathrm{BE}$ approach which starts from the BCS limit, insofar as the physics of the BE (compositeboson) region is much richer than its counterpart in the BCS limit. Specifically, the reverse approach enables one to perform approximations in the BE limit which would be a priori uncontrolled if one would start instead from the BCS limit. These approximations, which are necessarily more involved than those performed in the BCS limit, are nevertheless appropriate also to the BCS limit 8 In addition, it has been shown that even numerical approximation schemes starting from the BE region are surprisingly accurate also on the BCS side of the crossover, while the reverse is not true.6

The plan of the paper is as follows. In Section 2 we provide the general physical argument for the size shrinking of the composite bosons with increasing density in the "dilute limit", irrespective of dimensionality. In Section 3 we confront this general argument with the analytic zero-temperature mean-field results for a contact potential in the strong-coupling limit, both in three and two dimensions. Numerical results for two different finiterange potentials in three dimensions are also shown for comparison. Section 4 gives our conclusions.

\section{GENERAL ARGUMENT FOR THE SIZE SHRINKING OF THE COMPOSITE BOSONS}

We consider a system of fermions at zero temperature, mutually interacting via an (effective) attractive potential with a finite-range $r_{0}$. For the sake of comparison with the available analytic results in three and two dimensions, we disregard lattice effects and consider the system embedded in a homogeneous background (continuum case).

Composite bosons in isolation are defined when the associated two-body problem admits a bound state (zerodensity limit). At small (albeit finite) density, composite bosons are expected to retain their identity in the strong-coupling limit of the original fermionic attraction, i.e., when the binding energy of the associated two-body problem is much larger than the strength of the residual interaction between the composite bosons. Quite generally, this residual interaction has a dominant shortrange repulsive part due to Pauli principle, which is active among the constituent fermions as soon as the composite bosons overlap. This overlap, in turn, increases (on the average) when the size and/or the density of the composite bosons increase.

More precisely, we shall assume that $k_{F} a_{F}<<1$, where $a_{F}$ is the scattering length associated with the fermionic attraction, as well as $a_{F}>>r_{0}$. The former assumption represents a "diluteness" condition, while the second assumption is required to get a well-defined system of composite bosons, for which the Pauli repulsion overwhelms the attractive part of the bosonic potential originating from the finite-range fermionic attraction (see Ref. [8], footnote [36]). In this way, instabilities of the bosonic system (like the one recently pointed out in Ref. [9]) will be suitably avoided.

When the above conditions are satisfied, the composite bosons have a finite size and yet can be considered to be well-defined entities. We assume, therefore, that the standard results for the Bose gas can be used as far as the mutual interaction of the composite bosons is concerned, irrespective of their internal structure. To the internal structure we associate a pair wave function $\psi$ and a corresponding internal energy $\epsilon$, obtained from (minus) the expectation value $\left\langle\psi\left|H_{2}\right| \psi\right\rangle /\langle\psi \mid \psi\rangle$ of the two-fermion Hamiltonian $\mathrm{H}_{2}$ over the pair wave function (where $\mathrm{H}_{2}$ contains the reduced kinetic energy and the attractive two-fermion potential). Physically, $-\epsilon$ represents the energy required to form a composite boson in isolation with a given internal wave function $\psi$ for the pair of constituent fermions. We thus regard the energy $2 \mu$ required to add two fermions to the system as composed of two distinct contributions, namely, (i) the (negative of the) above internal energy $-\epsilon$ and (ii) the energy $\mu_{B}$ required to add eventually the composite boson to the system (with a finite density of composite bosons already present). On physical grounds, $\mu_{B}$ is related to the repulsive interaction between the composite bosons and depends on their size and density. Specifically, in three dimensions we take (we set $\hbar=1$ throughout)

$$
\mu_{B}=\frac{4 \pi n_{B} a_{B}}{m_{B}}
$$

from the standard theory of the "dilute" Bose gas, 10 where $n_{B}=n / 2$ is the bosonic density, $m_{B}=2 m$ is the mass of a composite boson in terms of the mass $m$ of the constituent fermions, and $a_{B}$ is the (positive) bosonic scattering length due to the repulsive interaction between the composite bosons. In two dimensions we take instead

$$
\mu_{B}=\frac{4 \pi n_{B}}{m_{B} \ln \left(\frac{1}{n_{B} r_{o}^{2}}\right)}
$$

from the theory of the two-dimensional "dilute" Bose gas, 11 where $r_{o}$ represents the range of the bosonic interaction. From dimensional considerations, both $a_{B}$ in Eq. (2.1) and $r_{o}$ in Eq. (2.2) should be proportional to the size $\xi_{\text {pair }}$ of the composite bosons, defined by the following functional:

$$
\xi_{\text {pair }}^{2}[\psi]=\frac{\int d \mathbf{r}|\psi(\mathbf{r})|^{2} \mathbf{r}^{2}}{\int d \mathbf{r}|\psi(\mathbf{r})|^{2}} .
$$

We set correspondingly $a_{B}=\alpha_{3} \xi_{\text {pair }}$ and $r_{o}=\alpha_{2} \xi_{\text {pair }}$ in three and two dimensions, in the order, where $\alpha_{3}$ and $\alpha_{2}$ are positive constants. In this way, the bosonic chemical 
potential $\mu_{B}=\mu_{B}\left(\xi_{\text {pair }}\right)$ becomes itself a function of $\xi_{\text {pair }}$.

We determine next the function $-\epsilon\left(\xi_{\text {pair }}\right)$ by minimizing $\left\langle\psi\left|H_{2}\right| \psi\right\rangle /\langle\psi \mid \psi\rangle$ in the subspace of the wave functions $\psi$ corresponding to a given value of $\xi_{\text {pair }}$. In this way, the equilibrium value $\xi_{\text {pair }}^{(o)}$ and the associated internal energy $\epsilon_{o}$ are obtained from the global minimum of $-\epsilon\left(\xi_{\text {pair }}\right)\left(\epsilon_{o}\right.$ coinciding with the binding energy of the associated twobody problem). Near this minimum we set

$$
\epsilon\left(\xi_{\text {pair }}\right)=\epsilon_{o}-A_{d}\left(\xi_{\text {pair }}-\xi_{\text {pair }}^{(o)}\right)^{2}
$$

where the positive constant $A_{d}$ depends in general on the dimensionality $d$. This approximate expression can be used when searching for the minimum of

$$
2 \mu\left(\xi_{\text {pair }}\right)=-\epsilon\left(\xi_{\text {pair }}\right)+\mu_{B}\left(\xi_{\text {pair }}\right)
$$

to determine the new equilibrium value $\bar{\xi}_{\text {pair }}$ in the presence of a finite (albeit small) density of composite bosons. From Eq. (2.1) we then obtain for this new equilibrium value in three dimensions:

$$
\bar{\xi}_{\text {pair }}=\xi_{\text {pair }}^{(o)}-\frac{2 \pi \alpha_{3}}{m_{B} A_{3}} n_{B}
$$

while from Eq. (2.2) we obtain in two dimensions:

$$
\bar{\xi}_{\text {pair }}=\xi_{\text {pair }}^{(o)}-\frac{\pi}{m_{B} A_{2} \xi_{\text {pair }}^{(o)}\left[\ln \left(\alpha_{2} \xi_{\text {pair }}^{(o)} n_{B}^{1 / 2}\right)\right]^{2}} n_{B}
$$

Note that the value of $\bar{\xi}_{\text {pair }}$ in the presence of a finite density of composite bosons is smaller than the value $\xi_{\text {pair }}^{(o)}$ for a composite boson in isolation, both in three and two dimensions. Physically, this shrinking is due to the fact that the decrease of the repulsive bosonic interaction (when the composite bosons contract) prevails over the reduction of the internal energy away from the original equilibrium value $\epsilon_{o}$.

In three dimensions, by inserting Eq. (2.6) into Eq. 2.4 $)$, the difference $\epsilon\left(\bar{\xi}_{\text {pair }}\right)-\epsilon_{o}$ is seen to decrease quadratically with increasing density. The leading-order correction to the internal energy $\epsilon$ is then quadratic in the density. This should be contrasted with the density dependence of the bosonic chemical potential, which is instead linear to the leading order. It can be further checked that adding to equation (1) for the bosonic chemical potential terms of higher order in the small parameter $n_{B}^{1 / 3} a_{B}$, will not modify expression (2.6) for the leading-order dependence of $\xi_{\text {pair }}$ on $n_{B}$ in the dilute limit. Both the bosonic chemical potential $\mu_{B}$ and the internal energy $\epsilon$ are thus self-consistently calculated by our approach to the leading order in the density.

To leading order, the binding energy $-2 \mu\left(\bar{\xi}_{\text {pair }}\right)$ for a composite boson embedded in the medium thus decreases linearly with the density, owing to the linear dependence of the bosonic chemical potential. This effect has been evidenced in different contexts, namely, for the Bose condensation of excitons in semiconductors 5 and, more recently 12 for the Bose condensation of atomic hydrogen. In the latter case, a linear reduction of the bosonic binding energy has been measured for increasing density, consistently with the general argument presented in this paper.

\section{COMPARISON WITH 3-D AND 2-D MEAN-FIELD RESULTS}

In this Section, we consider without loss of generality an attractive fermionic point-contact potential, for which the analytic solution of the BCS to BE crossover at the zero-temperature mean-field level is available, both in three 6 and two dimensions. We verify that this solution yields a decrease of $\xi_{\text {pair }}$ for increasing density, both in three and two dimensions, in generic agreement with the results of the previous Section. Specifically, in three dimensions we are able to recover the mean-field density dependence of $\xi_{\text {pair }}$ from the approach of the previous Section, by relating $a_{B}$ to $\xi_{\text {pair }}$ via the Born approximation. The analogous attempt fails, however, in two dimensions because the Born approximation (which is associated with mean field) strongly overestimates the scattering between composite bosons, thus disrupting the basic assumptions on which the approach of the previous Section rests.

We remark that the analytic results presented in this Section for a point-contact interaction describe also the general behaviour for a finite-range potential in the "dilute" composite-boson regime, which is defined by the two conditions $k_{F} a_{F}<<1$ and $a_{F}>>r_{0}$ previously mentioned. In this case, the range $r_{0}$ of the potential is much smaller than the two other length scales in the problem $\left(k_{F}^{-1}\right.$ and $\left.a_{F}\right)$, so that the mean-field equations for a finite-range potential get always mapped onto the mean-field equations for a point-contact interaction (with the same scattering length). The region where the size-shrinking effect is present (namely, the compositeboson region) coincides thus with the region where the behaviour of the point-contact interaction is "universal". It is just this coincidence which enables us to establish the size-shrinking effect as a general feature of the strongcoupling limit of the BCS-BE crossover.

\section{A. Three-dimensional case}

For a three dimensional point-contact potential, the low-energy fermionic two-body scattering can be conveniently regularized in terms of the scattering length $a_{F}$. At finite fermionic density, it is then possible to express all relevant physical quantities (such as the superconducting gap $\Delta$ and the chemical potential) in terms of the di- 
mensionless parameter $k_{F} a_{F}$. This can be explicitly verified for the analytic solution of Ref. [6] given in terms of the complete elliptic integrals. Since $k_{F} a_{F}<<1$ in the BE limit, physical quantities can further be expanded in powers of $k_{F} a_{F}$ in this limit.

In particular, for the pair coherence length one finds from the analytic solution of Ref. [6]:

$$
\xi_{\text {pair }}=\frac{a_{F}}{\sqrt{2}}\left[1-\frac{5}{6 \pi}\left(k_{F} a_{F}\right)^{3}\right]
$$

at the leading order in the bosonic density $n_{B}=$ $k_{F}^{3} /\left(6 \pi^{2}\right)$. Equation [8] has been obtained by first expanding equation (30) of Ref. [6] in powers of $1 / x_{0}$, by then inverting it to abtain $x_{0}$ in powers of $k_{F} a_{F}$, and by inserting the resulting expression for $x_{0}$ into the power expansion of equation (31) of Ref. [6] for $k_{F} \xi_{\text {pair }}$. In equation (8) (as well as in equation (31) of Ref. [6]) $\xi_{\text {pair }}$ is defined according to equation (2.3), with the BCS choice $\psi_{\mathrm{BCS}}(\mathbf{k})=\Delta /(2 E(\mathbf{k}))$ for the Fourier transform (with wave vector $\mathbf{k}$ ) of the pair wave function, where $E(\mathbf{k})=\sqrt{\left(\mathbf{k}^{2} /(2 m)-\mu\right)^{2}+\Delta^{2}}$ as usual.

Note from Eq. (3.1) that in the zero-density limit $\xi_{\text {pair }}$ equals the value $a_{F} / \sqrt{2}$ of the bound-state radius of a composite boson in isolation. Note also that $\xi_{\text {pair }} d e-$ creases linearly with increasing density of the composite bosons. The mean-field analytic solution for a pointcontact interaction thus confirms our general prediction of the size-shrinking effect discussed in the previous Section [cf. Eq. (2.6)].

By the same token, for the bosonic chemical potential one finds from the analytic solution of Ref. [6]:

$$
\mu_{B}=\frac{2 a_{F} k_{F}^{3}}{3 \pi m}=\frac{8 \pi n_{B} a_{F}}{m_{B}} .
$$

Comparison with Eq.(2.1) suggests then to identify $a_{B}=$ $2 a_{F}$ in the BE (strong-coupling) limit. This result, which was also obtained for the same model system within the fermionic T-matrix approximation in the normal state (i.e., above the superconducting critical temperature), 3 , identifies $k_{F} a_{F}$ with $\left(3 \pi^{2} / 4\right)^{1 / 3}$ times the "gas parameter" $n_{B}^{1 / 3} a_{B}$ for a "dilute" Bose gas.

Recall further that the general mapping procedure from the original fermionic system onto the effective bosonic system in the strong-coupling limit (as described in Ref. [4]) provides in three dimensions the value

$$
v(0)=\frac{4 \pi a_{F}}{m}
$$

for the strength of the "bare" bosonic potential (with all wave vectors and Matsubara frequencies set to zero). One then verifies that the Bogoliubov result

$$
\mu_{B}=n_{B} v(0)
$$

is retrived by the expression (3.2) 13
It is interesting to show that not only the generic linear density dependence but also the coefficients of the expression (3.1) can be reproduced by the variational principle of the previous Section, provided we take for $\alpha_{3}$ the value $2 \sqrt{2}$ (as determined from Eqs. (3.2), (3.1), and (2.1) to leading order in the density) and we assume the BCS form $\psi_{\mathrm{BCS}}(\mathbf{k})=\Delta /(2 E(\mathbf{k}))$ for the Fourier transform of the pair wave function. To this end, it is convenient to express initially $\left\langle\psi\left|H_{2}\right| \psi\right\rangle /\langle\psi \mid \psi\rangle$ and $\mu_{B}$ as functions of the dimensionless variable $x_{o}=\mu / \Delta$ instead of $\xi_{\text {pair }}$, to minimize then the resulting expression for $2 \mu$ with respect to $x_{o}$, and to use eventually the functional relation between $x_{o}$ and $\xi_{\text {pair }}$ (as determined in Ref. [6]) to obtain $\xi_{\text {pair }}$ in terms of $k_{F} a_{F}$. In the BE limit, where $x_{o}<0$ and $\left|x_{o}\right|>>1$, this relation reads:

$$
k_{F} \xi_{\text {pair }}=\frac{1}{\sqrt{2}}\left(\frac{3 \pi}{16 x_{o}^{2}}\right)^{1 / 3}\left(1-\frac{1}{4 x_{o}^{2}}\right)
$$

to the leading significant orders. One finds:

$$
\begin{aligned}
\frac{\left\langle\psi_{\mathrm{BCS}}\left|H_{2}\right| \psi_{\mathrm{BCS}}\right\rangle}{\left\langle\psi_{\mathrm{BCS}} \mid \psi_{\mathrm{BCS}}\right\rangle} & =\frac{k_{F}^{2}}{2 m}\left(\frac{16}{3 \pi}\right)^{2 / 3}\left[2\left|x_{o}\right|^{4 / 3}-4 b\left|x_{o}\right|^{2 / 3}\right. \\
& \left.+\frac{5}{8}\left|x_{o}\right|^{-2 / 3}-\frac{5}{8} b\left|x_{o}\right|^{-4 / 3}\right]
\end{aligned}
$$

where we have set $b=(3 \pi / 16)^{1 / 3}\left(k_{F} a_{F}\right)^{-1}$, while $\mu_{B}=$ $\mu_{B}\left(x_{o}\right)$ is given by Eq. (2.1) with $a_{B}=2 \sqrt{2} \xi_{\text {pair }}$ and with $\xi_{\text {pair }}=\xi_{\text {pair }}\left(x_{o}\right)$ given by Eq. 3.5 .

At the leading order, only the first two terms within brackets in Eq. (3.6) are relevant to the expression of $2 \mu$, whose mimimum is thus located at $\left|\bar{x}_{o}\right|=b^{3 / 2}$. At the next significant order, all terms within brackets in Eq. 3.6) and the leading term in Eq. (3.5) are relevant to the expression of $2 \mu$, yielding the new minimum at

$$
\left|\bar{x}_{o}\right|=b^{3 / 2}\left(1-\frac{9}{64 b^{3}}\right) .
$$

Inserting Eq. (3.7) into Eq. (3.5) one recovers eventually the expression (3.1) for $\xi_{\text {pair, as anticipated. The fact }}$ that equation (8), obtained by the mean-field solution, can be reproduced with the correct numerical coefficients by the minimization procedure of the previous Section (when specialized to the BCS choice for the pair wave function $\psi$ ), can be regarded as quite a compelling check on the validity of our general argument of Section 2 and of its underlying assumptions.

Finally, it is interesting to examine the behaviour of $\xi_{\text {pair }}$ as a function of the density for a finite-range potential, for which the BCS-BE crossover driven by the density becomes possible 2. As in Ref. [2], we consider the separable Nozières-Schmitt-Rink (NSR) potential $V\left(k, k^{\prime}\right)=V\left(1+k^{2} / k_{0}^{2}\right)^{-1 / 2}\left(1+k^{\prime 2} / k_{0}^{2}\right)^{-1 / 2}($ with $k=|\mathbf{k}|)$, and the non-separable Gaussian potential $V\left(\mathbf{k}, \mathbf{k}^{\prime}\right)=V \exp \left(-\left|\mathbf{k}-\mathbf{k}^{\prime}\right|^{2} / k_{0}^{2}\right)(V<0$ in both cases $)$. For these potentials, an analytic solution for the BCS-BE crossover is lacking, even at the mean-field level. We have 
therefore solved numerically the coupled equations for the gap function $\Delta(k)$ and the chemical potential $\mu$. The pair-coherence length $\xi_{\text {pair }}$ has been then determined by using the Fourier transform of equation (3), with the BCS choice for the pair wave function $\psi(k)=\Delta(k) /(2 E(k))$. Results are shown in Figure 1, which confirm the presence of the size-shrinking effect in the "universal" compositeboson region. Note, however, that the size-shrinking effect gradually disappears by increasing further the density: $\xi_{\text {pair }}$ reaches a minimum at $k_{F} \sim k_{0}$ and then starts increasing with the density. The eventual increase of $\xi_{\text {pair }}$ with $k_{F}$, when $k_{F}>>k_{0}$, is a characteristic feature of a finite-range potential, as it can be readily verified from the asymptotic expressions (4) and (5) of Ref. [2] for the gap $\Delta\left(k_{F}\right)$ in the limit $k_{F}>>k_{0}$. In this limit, as soon as the system enters the BCS regime, we may use the BCS result $\xi_{\text {pair }} \sim k_{F} / \Delta\left(k_{F}\right)$ to obtain $\xi_{\text {pair }} \sim k_{F}^{-1} \exp \left(\frac{k_{F} A}{|V| m k_{0}^{2}}\right)$, where $A$ is a numerical factor different for the NSR and Gaussian potentials. For both potentials, $\xi_{\text {pair }}$ is seen to increase exponentially with $k_{F}$ for large $k_{F}$, as far as $k_{0}$ is finite. The competition between this asymptotic behaviour of $\xi_{\text {pair }}$ when $k_{F}>>k_{0}$ and the initial size-shrinking when $k_{F}<<k_{0}$, leads then to a minimum of $\xi_{\text {pair }}$ when $k_{F} \sim k_{0}$.

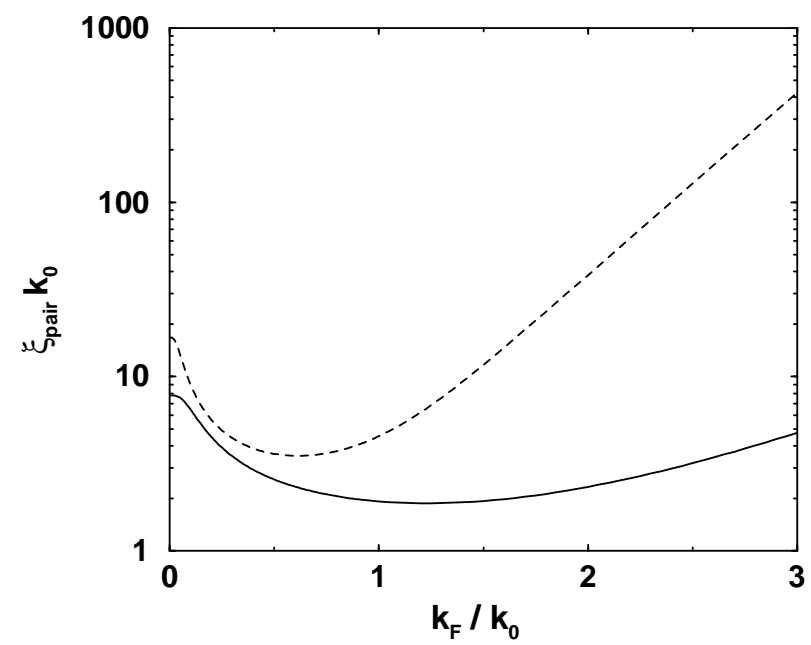

FIG. 1. Pair coherence length (in units of the characteristic length $k_{0}^{-1}$ of the finite-range fermionic potential) vs $k_{F} / k_{0}$, for the NSR (full line) and Gaussian (dashed line) potentials. [A typical value of $|V|\left(=1.1 V_{c}\right)$ above the critical value $V_{c}$ for the existence of a bound state in three dimensions has been considered for both potentials.]

\section{B. Two-dimensional case}

In two dimensions, the zero-temperature mean-field expression for $\xi_{\text {pair }}$ is reported in Appendix B of Ref. [6] in terms of the available analytic solution. In particular, at the leading significant order in the BE limit one finds:

$$
\xi_{\text {pair }}^{2}=\frac{2}{3 m \epsilon_{o}}\left[1-\frac{38}{15}\left(\frac{4 \pi n_{B}}{m_{B} \epsilon_{o}}\right)\right] .
$$

For given value of $\epsilon_{o}, \xi_{\text {pair }}$ is thus seen to decrease linearly with the density in the BE limit. One also finds for the bosonic potential:

$$
\mu_{B}=\frac{8 \pi n_{B}}{m_{B}}
$$

which is proportional to the density but independent from the bosonic size. Note from Eq. (3.9) that the Bogoliubov result $\mu_{B}=n_{B} v(0)$ is retrieved by the mean-field calculation even in two dimensions, since the strength $v(0)$ of the "bare" bosonic potential is given by

$$
v(0)=\frac{8 \pi}{m_{B}},
$$

as it can be explicitly verified by applying the prescriptions of Ref. [4] to the fermion-boson mapping in the two-dimensional case.

The mean-field expressions (3.8) and (3.9) differ from the "dilute" Bose gas expressions (2.7) and (2.2), respectively, as they lack the logarithmic term in the denominator. Notwithstanding the decrease of the size of the composite bosons for increasing density obtained by Eq. (3.8), the two-dimensional mean-field results appear thus to contradict the picture of Section II for a "dilute" gas of composite bosons. In particular, if one would use the Bogoliubov expression (3.9) to implement the argument of Section II, the failure of the strength of the "bare" bosonic potential in two dimensions to depend on the size of the composite bosons would not make it energetically convenient to shrink the bosonic size at finite density. The size shrinking obtained by Eq. (3.8) is therefore not consistent with the general argument developed in Section II.

We attribute the difference between the mean-field and the "dilute" gas results to the poor treatment of the boson-boson scattering within the zero-temperature mean field, which rests on the Born approximation as the form (3.9) of the bosonic chemical potential implies. Let us, in fact, analyze the above results in terms of the out comes of potential scattering theory in two dimensions. 14 The Born approximation gives for the (dimensionless) low-energy scattering amplitude $f_{\mathrm{Born}}^{(2)} \sim-m v(0)$, where $v(0) \sim v_{o} r_{o}^{2}$ is typically proportional to the average strength $v_{o}$ and range $r_{o}$ of the potential. The exact low-energy result for the two-dimensional scattering amplitude $f^{(2)} \sim-1 / \ln \left(k r_{o}\right)$, on the other hand, is independent of $v_{o}$ and vanishes for vanishing wave vector $(k \rightarrow 0)$. In two dimensions, therefore, the effect of summing an infinite number of repeated scatterings drastically modifies the functional dependence of the scattering amplitude on the strength and range of the potential, and the perturbation theory for the scattering amplitude breaks down 15 In contrast, in three dimensions $f_{\text {Born }}^{(3)} \sim-m v(0) \sim-a_{\text {Born }}$ where $v(0) \sim v_{o} r_{o}^{3}$ and $a_{\text {Born }}$ is the Born scattering length, whereas $f^{(3)} \sim-a$ is the exact low-energy result ( $a$ here being the full scattering length). That is, in three dimensions going from 
the Born approximation to the exact low-energy result merely changes the numerical value of the scattering length.

For the composite bosons we are specifically interested in, the strength $v_{o}$ must be proportional to the binding energy $\epsilon_{o}$ for dimensional reasons, and the only available length scale is proportional to $\left(m \epsilon_{o}\right)^{-1 / 2}$. This yields $v(0) \sim \epsilon_{o}\left(m \epsilon_{o}\right)^{-d / 2}$, which corresponds to Eqs. (3.3) and (3.10) for $d=3$ and $d=2$, respectively, implying that the characteristic strength and range of the potential cannot be independently varied for the composite bosons. It is this feature which, in turn, makes $v(0)$ independent from the size of the composite bosons in two dimensions. The Born approximation to the two-dimensional scattering amplitude for the composite bosons, therefore, not only lacks the functional form of the exact low-energy result, but even fails to yield any dependence on the size of the composite bosons whatsoever.

From the above considerations, it is thus evident that in two dimensions the scattering rate between the composite bosons is overestimated within the Born approximation with respect to the true low-density result, in such a way that the "dilute limit" cannot be achieved at fixed density. Consequently, the physical picture adopted in Section II of a system of well-defined weakly interacting bosonic entities is bound to break down within mean field.

\section{CONCLUDING REMARKS}

In this paper, we have discussed the size shrinking of composite bosons as a general physical result occurring in the "dilute limit". We have also verified that this general result is correctly reproduced by the zero-temperature mean-field treatment in three but not in two dimensions. We have related the failure in two dimensions to the break down of the Born approximation at low energy. In addition, we have noted a peculiar relation between the effective strength and range of the potential acting between composite bosons.

For the three-dimensional case, the relevance of including the mutual interaction between the composite bosons in the strong-coupling (BE) limit has been emphasized some time ago in Refs. [5] and [16]; more recently, the significance of treating the scattering between the composite bosons beyond the Born approximation has been addressed in Ref. [8]. We have argued here, however, that the failure to account for the effects of the bosonic interaction is more severe in two than in three dimensions, because the Born approximation breaks completely down in two dimensions. By the same token, we also expect that the boundary in the phase diagram between the Bose-Einstein and crossover regions, discussed in Ref. [2] within mean field, should be significantly modified by a proper treatment of the bosonic interaction, along the lines recently developed for the three-dimensional case. 8
It is further clear that the criticisms recently raised to the fermionic T-matrix approximation when applied to the normal phase in three dimensions (which in the strong-coupling limit has been proved to reduce to the Born approximation as far as the scattering between composite bosons is concerned $\$$ ) are even more appropriate for the two-dimensional case where the Born approximation fails completely. This remark makes it somewhat questionable the use of the fermionic (sejf-consistent) Tmatrix approximation in two dimensions, 17 to describe the tendency toward the formation of preformed pairs above the superconducting critical temperature, at least as the strong-coupling (bosonic)limit is approached.

\section{ACKNOWLEDGMENTS}

One of us (P.P.) gratefully acknowledges receipt of a postdoctoral research fellowship from the Italian INFM under contract PRA-HTCS/96-99.

${ }^{1}$ F. Pistolesi and G.C. Strinati, Phys. Rev. B49, 6356 (1994).

2 N. Andrenacci, A. Perali, P. Pieri, and G.C. Strinati, condmat/9903399, to appear in Phys. Rev. B.

${ }^{3}$ R. Haussmann, Z. Phys. B91, 291 (1993).

${ }^{4}$ F. Pistolesi and G.C. Strinati, Phys. Rev. B53, 15168 (1996).

${ }^{5}$ A similar relation between the chemical potentials of the constituent fermions and of the composite bosons has been discussed in the paper by L.V. Keldysh and A.N. Kozlov [Sov. Phys. JETP 27, 521 (1968)], in the context of the Bose condensation of excitons in semiconductors.

${ }^{6}$ M. Marini, F. Pistolesi, and G.C. Strinati, Eur. Phys. J. B1, 151 (1998).

${ }^{7}$ M. Randeria, J.-M. Duan, L.-Y. Shieh, Phys. Rev. B41, 327 (1990).

${ }^{8}$ P. Pieri and G.C. Strinati, cond-mat/9811166.

${ }^{9}$ G. Röpke, A. Schnell, P. Schuck, and P. Nozières, Phys. Rev. Lett. 80, 3177 (1998).

${ }^{10}$ Cf., e.g., A.L. Fetter and J.D. Walecka, Quantum Theory of Many-Particle Systems (McGraw-Hill, New York, 1971), Section 22.

${ }^{11}$ D.S. Fisher and P.C. Hohenberg, Phys. Rev. B37, 4936 (1988).

12 T.C. Killian, D.G. Fried, L. Willmann, D. Landhuis, S.C. Moss, T.J. Greytak, and D. Kleppner, Phys. Rev. Lett. 81, 3807 (1998); D.G. Fried, T.C. Killian, L. Willmann, D. Landhuis, S.C. Moss, D. Kleppner, and T.J. Greytak, Phys. Rev. Lett. 81, 3811 (1998).

${ }^{13}$ It can be also verified that the hydrodynamic relation $\mu_{B}=$ $m_{B} s^{2}$ (where $s$ is the sound velocity) noted by Keldysh and Kozlov in Ref. [5], is recovered from the analytic solution of Ref. [6] both in three and two dimensions. 
${ }^{14}$ S. K. Adhikari, Am. J. Phys. 54, 362 (1986).

15 See, e.g., L.D. Landau and E.M. Lifshitz, Quantum Mechanics (Pergamon Press, Oxford, 1958), p.154.

${ }^{16}$ P. Nozières and S. Schmitt-Rink, J. Low. Temp. Phys. 59, 195 (1985).

${ }^{17}$ B. Kyung, E.G. Klepfish, and P.E. Kornilovitch, Phys. Rev. Lett. 80, 3109 (1998).

18 J.R. Engelbrecht and A. Nazarenko, cond-mat/9806223. 